\title{
Quality of Life among Caregivers of Lower-Income Cancer Patients: A Single-Institutional Experience in India and Comprehensive Literature Review
}

\author{
Vishal Vashistha ${ }^{1}$, Rosemary Poulose ${ }^{2}$, Chandrashekhar Choudhari ${ }^{3}$, Sukhdeep \\ Kaur $^{4}$, Anant Mohan ${ }^{5}$
}

${ }^{1}$ Fellow, Department of Hematology and Oncology, Duke University Medical Center, Durham, NC, U.S.A. ${ }^{2}$ Resident, Department of Pulmonary Medicine and Sleep Disorders, All India Institute of Medical Sciences, Delhi, India. ${ }^{3}$ Resident, Department of Pulmonary Medicine and Sleep Disorders, All India Institute of Medical Sciences, Delhi, India. ${ }^{4}$ Resident, Department of Internal Medicine, St. Joseph's Hospital and Medical Center, Phoenix, AZ, U.S.A. ${ }^{5}$ Professor, Department of Pulmonary Medicine and Sleep Disorders, All India Institute of Medical Sciences, Delhi, India.

\begin{abstract}
Objectives: Quality of life (QOL) among caregivers of cancer patients is often diminished. For lower-income caregivers, the deterioration in QOL may be of greater impact. We aimed to evaluate QOL among lower-income cancer caregivers in Delhi, India and to comprehensively review similar investigations conducted internationally. Methods: A Hindi-version of the Caregiver Quality of Life Cancer (CQOLC) index was administered to 89 caregivers of lung cancer patients. Bivariate analyses were employed to evaluate associations between baseline demographics and CQOLC index scores. A systematic review of PubMed, EMBASE, and PsychInfo was undertaken. Results: Reduced QOL was observed for caregivers residing in homes earning less than our center's median annual income per capita $(\mathrm{p}<0.01)$ and for caregivers providing aid for 4 months or longer $(\mathrm{p}<0.01)$. The burden of caregiving contributed most to summative index scores $(\mathrm{p}<0.01)$. Eleven studies were selected by systematic review. Lower-income caregivers in Asia and the Middle East experience reduced QOL and increased burden. Available evidence does not suggest that lower-income caregivers in Europe and North America share worse QOL. Conclusion: Lower-income caregivers suffer loss in QOL, particularly in the developing world. Resourceful interventions are warranted to mitigate burden for this underappreciated population.
\end{abstract}

Keywords: Cancer Caregiver- Caregiver Burden- underserved populations- quality of life

Asian Pac J Cancer Care, 4 (3), 87-93

\section{Introduction}

\section{Background}

Caregivers of patients suffering from cancer unfortunately experience deterioration in quality of life (QOL) [1-2]. The physical, emotional, social, and financial concerns associated with caring for a cancer patient may lead to both short-term and long-term difficulties for caregivers [3-4]. Commonly, these concerns are unnoticed by medical providers as focus is directed towards treating patients for their malignancies. For caregivers of lower socioeconomic status, the burden may be considerably more overwhelming.

Previous work has conveyed that caregivers are
Submission Date: 02/06/2019 Acceptance Date: 04/02/2019

often required to spend a substantial percentage of their own savings while providing aid to their loved ones [5]. The loss in caregiver income and reduction in savings is regularly excluded in estimates quantifying the costs of cancer care [6]. Specifically, lower-income caregivers and those who are responsible for other dependents are at high-risk for losing a significant portion of their financial reserves. Furthermore, the limited accessibility to support, information, and medical technology may lead to greater duress for lower-income caregivers.

In India, lung cancer is the most common cause of cancer-related mortality for men and the 7 th most common cause for women [7]. Due to increasing use of cigarettes and bidis by the illiterate and lesser-

Corresponding Author:

Dr. Vishal Vashistha

Fellow, Department of Hematology and Oncology, Duke University Medical Center, Durham, NC, U.S.A

Email: vishal.vashistha@duke.edu 
educated, the incidence of lung cancer is expected to rise, particularly among lower-income patients [8]. The rising incidence of lung cancer will unfortunately lead to more widespread emotional and financial stress on family caregivers of lower-socioeconomic status. Consequently, an investigation identifying the specific lower-income caregivers who may struggle most while aiding lung cancer patients is warranted to design future resourceful interventions for this underappreciated population.

Herein, we report the results of a single-institutional, cross-sectional assessment of QOL among lower-incomes caregivers of patients with advanced lung cancer residing within Delhi, India or the National Capital Region (NCR). Additionally, we comprehensively reviewed previous studies that assessed QOL among lower-income caregivers of cancer patients in either the developed or developing world. Possible interventions to mitigate reduction in QOL were identified.

\section{Materials and Methods}

\section{Institutional Experience}

A cross-sectional, survey-based investigation among family caregivers who accompanied patients to the Lung Cancer Clinic (LCC) at The All India Institute of Medical Sciences (AIIMS)-Delhi between fall 2016 and spring 2017 was conducted. The LCC at AIIMS-Delhi is a major public referral center serving lower-income patients suffering from lung cancer in Delhi, India and the NCR. Permission to complete this study was granted by the Institutional Ethics Board at AIIMS-Delhi.

\section{Study Participants}

Eligible caregivers were required to be (1) related to patients who had been histologically-diagnosed with either non-small cell or small cell lung cancer, (2) over 18 years of age, (3) able to communicate in either Hindi or English, and (4) unpaid for their services. At the time of caregiver assessment, patients were either being evaluated for treatment or were actively undergoing cycles of cytotoxic chemotherapy. Attempts were undertaken to survey caregivers who were primarily assisting patients at home.

\section{Measurement}

A Hindi-version of the Caregiver Quality of Life Cancer (CQOLC) index was administered to all eligible caregivers. The CQOLC index is a 35-item assessment comprising 4 major subdomains [9-10]. These subdomains include burden (10 items), disruptiveness (7 items), positive adaptation ( 7 items), and financial concerns (3 items). The index also includes 8 additional items that are not associated with any of the major subdomains. Caregivers respond to each item using a Likert scale ranging from 0 to 4 , with 4 indicating that an individual item resonates strongly with the caregiver. Responses for 27 items contribute as positive additions to the caregiver's overall score while 8 responses contribute as scoring deductions. Higher scores indicate worse QOL among caregivers.

\section{Data Collection}

Eligible caregivers were ushered to a private setting during a scheduled appointment for their respective patients. A copy of the Hindi-version of the CQOLC index was provided by a trained researcher. Assistance was offered to partially-illiterate caregivers during the completion of the surveys. Unanswered items were scored as "zero". Surveys with more than 7 unfilled responses $(>20 \%)$ were excluded from analysis. Attempts to obtain demographic data were undertaken at the time of caregiver assessment.

\section{Statistical Analysis}

Microsoft Excel version 2016 was used to analyze the data. Descriptive statistics were calculated to evaluate baseline demographics of the included caregivers. Continuous demographic variables were converted to categorical variables based on the computed means and medians. Two-tailed t-tests or one-factor analysis of variance (ANOVA) were conducted to examine associations between demographic categorical variables and mean CQOLC index scores. Standard deviations were calculated with each mean score. Statistical significance was confirmed for $\mathrm{p}<0.05$. As arbitrarily defined by previous work, a clinically meaningful difference (CMD) required subgroup scores to differ by 0.5 standard deviations in magnitude.[2] The mean index scores for each subdomain were computed individually, and these scores were compared using ANOVA.

\section{Comprehensive Literature Review}

A systematic review of literature that evaluated QOL among lower-income caregivers of cancer patients was completed. The systematic review was performed in accordance with PRISMA guidelines [11]. A search of PubMed, EMBASE, and PsychInfo databases was conducted using the following phrase: "cancer AND caregiver AND (socioeconomic OR lower-income OR poverty OR poor)".

\section{Study Inclusion Criteria}

Investigations that either detailed QOL solely among lower-income caregivers for cancer patients or reported the association between QOL and socioeconomic status were selected. Selected studies were required to (1) be published articles, (2) assess QOL by administering a standardized survey to cancer caregivers, (3) query caregivers over 18 years of age, (4) and evaluate QOL among caregivers within 6 months of cancer treatment for their respective patients. Studies that combined the results of caregivers and cancer patients were excluded.

\section{Data Synthesis}

Studies were grouped based on geographical location. The methodology and results from each selected study were reviewed in detail. The differences in QOL assessment scores between lower-income caregivers and caregivers with more financial flexibility were extracted. The QOL among lower-income caregivers from different regions of the world were compared. 
Quality Assessment

The National Heart, Lung, and Blood Institute's Quality Assessment Tool for Observational Cohort and Cross-Sectional Studies was used to assess quality for each selected publication [12]. This 14-item quality assessment tool evaluates study methodology and population sample for bias. Studies were rated as "good", "fair", and "poor" following review.

\section{Results}

Institutional Experience

In total, the survey data from 89 eligible caregivers were analyzed. All 89 caregivers provided their age, sex, relation to patient, and duration of care. A lesser number of caregivers provided their income, education, and living background. The mean age $+/$ - standard deviation was $37.8+/-9.2$ years while the median annual income per capita was 175,000 Rs. (\$2516.67 US). The demographics of included caregivers are described in Table 1.

\section{Summative CQOLC Index Scores}

The overall mean CQOLC score was 29.4 +/- 19.7, providing a coefficient of variation of $67.0 \%$. Higher scores were observed for caregivers who provided aid for more than 4 months compared to those who provided aid for shorter periods of time $(\mathrm{p}<0.01)$ and for caregivers living within residences earning less than 175,000 Rs. annually per capita compared to those living within

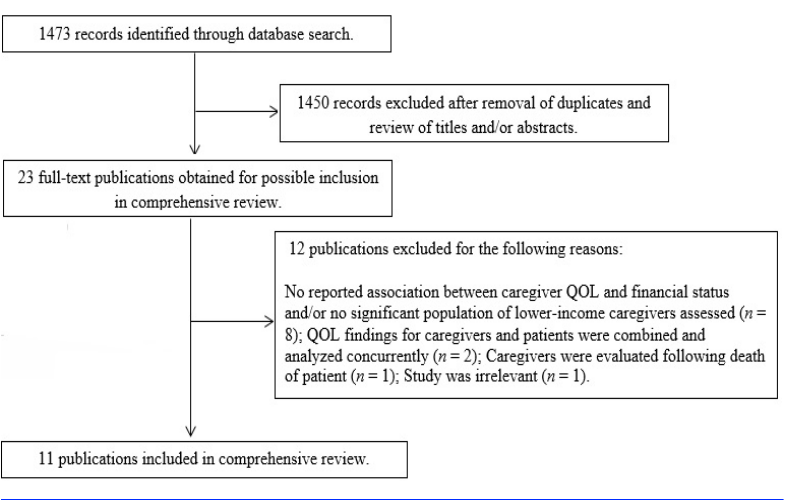

Figure 1. Study Selection Flowchart for Comprehensive Review

residences earning more per capita $(\mathrm{p}<0.01)$. In addition to statistical significance, a CMD was observed between each of the above subgroups. The associations between baseline demographic variables and caregiver scores are conveyed in Table 1.

\section{Subdomain Scores}

Among the 4 subdomains assessed within the CQOLC index, the highest mean score per item was observed for questions related to burden $(\mathrm{p}<0.01)$. The mean score for each individual subdomain is conveyed in Table 2. Responses to item 31, "It upsets me to see my loved one deteriorate", contributed the most positive additions to overall CQOLC index scores. Responses to item 10, "I

Table 1. Baseline Caregiver Demographics and Mean CQOLC Index Scores

\begin{tabular}{|c|c|c|c|}
\hline \multirow{2}{*}{$\begin{array}{l}\text { Demographic Variable } \\
\text { Age (89 Responses): }\end{array}$} & \multirow[t]{2}{*}{ Number of Caregivers } & \multicolumn{2}{|c|}{ Mean CQOLC Index Scores } \\
\hline & & & \\
\hline Caregiver under 40 years: & $55(61.8 \%)$ & $31.9+/-18.8$ & $\mathrm{p}=0.13$ \\
\hline Caregiver 40 years or older: & $34(38.2 \%)$ & $25.4+-20.8$ & \\
\hline \multicolumn{4}{|l|}{ Sex (89): } \\
\hline Female Caregiver: & $12(13.5 \%)$ & $35.5+/-28.1$ & $\mathrm{p}=0.25$ \\
\hline Male Caregiver: & $77(86.5 \%)$ & $28.5+/-18.2$ & \\
\hline \multicolumn{4}{|l|}{ Caregiver Relation (89): } \\
\hline Spouse of patient: & $9(10.1 \%)$ & $30.8+/-31.0$ & $\mathrm{p}=0.69$ \\
\hline Child or Sibling of patient: & $70(78.7 \%)$ & $28.6+/-17.3$ & \\
\hline Other family relative: & $10(11.2 \%)$ & $34.2+/-24.9$ & \\
\hline \multicolumn{4}{|l|}{ Duration of Care (89): } \\
\hline 4 months or less: & $50(56.2 \%)$ & $24.3+/-18.0$ & $\mathrm{p}<0.01^{*}$ \\
\hline Greater than 4 months: & $39(43.8 \%)$ & $36.0+/-20.1$ & \\
\hline \multicolumn{4}{|l|}{ Caregiver Education (44): } \\
\hline Did not complete high school: & $30(68.1 \%)$ & $38.7+/-22.7$ & $\mathrm{p}=0.21$ \\
\hline Completed high school: & $14(31.8 \%)$ & $29.4+/-22.5$ & \\
\hline \multicolumn{4}{|l|}{ Living Background (42): } \\
\hline Village: & $24(57.1 \%)$ & $39.9+/-22.6$ & $\mathrm{p}=0.11$ \\
\hline City: & $18(42.9 \%)$ & $28.3+/-24.4$ & \\
\hline \multicolumn{4}{|l|}{ Annual Income per Capita (38) } \\
\hline Under 175,000 Rs. per year: & $19(50.0 \%)$ & $48.4+/-22.0$ & $\mathrm{p}<0.01 *$ \\
\hline Over 175,000 Rs. per year: & $19(50.0 \%)$ & $21.1+/-19.5$ & \\
\hline
\end{tabular}

*A clinically meaningful difference was also met. Rs, Rupees. 
Table 2. Mean Scores and Standard Deviations for each Subdomain of the CQOLC Index

\begin{tabular}{lcc}
\hline Subdomain & Mean Score per Item & \\
\hline Burden (10 items): & $1.5+/-2.0$ & $\mathrm{p}<0.01$ \\
Disruptiveness (7 items): & $0.8+/-1.9$ & \\
Positive Adaption (7 items): & $1.1+/-2.5$ & \\
Financial Concerns (3 items): & $1.3+/-1.5$ \\
Undefined Subdomain (8 items): & $-0.3+/-3.0$ \\
\hline
\end{tabular}

have more of a positive outlook on life since my loved one's illness", contributed the least negative additions to overall index scores.

\section{Comprehensive Literature Review}

The search of included databases retrieved 1473 records for review. Following review of titles and/or abstracts, 1450 records were excluded and 23 full-text articles were obtained for detailed assessment. Eleven studies were selected after review of the full-text publications [13-23]. The study selection protocol is delineated in Figure 1.

Seven studies compared standardized assessment scores among caregivers based on tiered income levels [15-20, 22]. Two studies compared scores based on employment status [16-23]. Vahidi et al. compared scores based on personal views of financial stability among caregivers [21]. Lastly, Duggleby et al. assessed outcomes solely among rural caregivers [13]. The most commonly used assessment was the Zarith Burden Interview (ZBI), which was included in $4(36.3 \%)$ selected studies [14-16-21, 23]. The population of caregivers, standardized assessments, results, and quality ratings of each study are detailed in Table 3.

\section{Quality Ratings}

The quality of 7 studies (63.6\%) was rated as "good" while that of 4 studies (36.4\%) was rated as "fair". Among those rated as "fair", 4 publications did not report a statistical justification for the number of included caregivers, and 3 publications surveyed a relatively heterogeneous population of cancer caregivers.

\section{Discussion}

The findings from this single-institutional, crosssectional study convey the QOL deterioration that is evident among lower-income Indian caregivers of cancer patients. Worse CQOLC index scores were observed among caregivers who have been providing aid for longer durations of time and those living within residences earning a lesser amount of total income. Trends toward worse CQOLC index scores were observed among caregivers under 40 years of age and those residing in a village. A substantial variation in total index scores was observed.

Interestingly, the index items related to financial concern did not contribute most to overall scores. Rather, the burden subdomain of the CQOLC index was associated with the highest mean score per item. Lower- income caregivers may face greater burden because of limited accessibility to services and support at home. In this study, the two items related to caregiver burden that received the highest responses were associated with the need for caregivers to be available to tend to patients at all times. Further studies exploring the specific tasks that most impact caregiver burden are warranted. A foundational understanding of the burden experienced by lower-income caregivers could lead to interventions that improve overall QOL while conserving resources.

Our cross-sectional study is limited by a small number of female caregivers and confounding variables that were not independently assessed. These variables include distance travelled to reach appointments at the AIIMS LCC, the available support from other relatives, and the caregivers' communal social structures. Lastly, though attempts were undertaken to survey primary caregivers, the CQOLC index could only be administered to caregivers who traveled with patients to at least one appointment.

\section{Lower-Income Cancer Caregivers in Europe and North America}

One study conducted in Europe and 3 studies conducted in North America were identified by systematic review [13, 16, 18, 19]. In the Netherlands, Nijboer et al. administered the Caregiver Reaction Assessment (CRA) scale at three-month intervals to caregivers for colorectal cancer patients undergoing treatment at 1 of 10 regional centers $m$ [18]. Caregivers of lower socioeconomic status reported greater variability among changes in their financial problems, schedules, and self-esteem over time. No large differences in effect size were identified among caregivers of varying socioeconomic groups. In Canada, Duggleby et al. evaluated hope, grief, and mental and physical health among rural female caregivers of stage IV cancer patients [13]. The annual combined income was less than CAD $\$ 29,999.00$ for $31.9 \%$ of patients. Based on responses to the Short Form Health Survey Version $2(\mathrm{SF} 12 \mathrm{v} 2)$, the mental and physical subscores for rural female caregivers were near the 25 th percentile of the general US population. The authors did not report associations between QOL and varying levels of socioeconomic status. In the U.S.A., Goldstein et al. found no differences in ZBI scores based on household income among caregivers of terminally-ill cancer patients admitted to an inpatient hospice facility in Connecticut [16]. Rather, increasing burden was observed for younger caregivers and those sharing limited social networks. Oberst et al. assessed caregivers of patients undergoing 
Table 3. Selected Publications Evaluating QOL among Lower-Income Caregivers

\begin{tabular}{|c|c|c|c|c|}
\hline Study & Country & Population and Assessment & Findings & Quality \\
\hline Duggleby et al. 2014 & Canada & $\begin{array}{l}\text { Population: } 122 \text { female caregivers of cancer patients } \\
\text { living in a postal code within a rural region. } 57 \\
(46.7 \%) \text { caregivers maintained a household income } \\
\text { under CAD } \$ 39,999.00 \text {. } \\
\text { Assessments: GSES and SF-12v2. The grief and } \\
\text { hope among caregivers were also evaluated. }\end{array}$ & $\begin{array}{l}\text { Rural female caregivers in Canada were found to } \\
\text { have SF-12v2 mental and physical subscores that } \\
\text { were equivalent to or below the } 25 \text { th percentile of } \\
\text { scores for the general population in the U.S.A. The } \\
\text { level of hope among caregivers was proportional } \\
\text { to their general self-efficacy scores. }\end{array}$ & Good \\
\hline Goldstein et al. 2014 & U.S.A. & $\begin{array}{l}\text { Population: } 206 \text { caregivers of cancer patients } \\
\text { admitted to an inpatient hospice facility in } \\
\text { Connecticut. Subjects were stratified by annual } \\
\text { household income. } \\
\text { Assessment: ZBI. }\end{array}$ & $\begin{array}{l}\text { No difference in QOL was observed among } \\
\text { caregivers from homes earning more than US } \\
\$ 50,000.00 \text { compared to those from homes earning } \\
\text { less than US } \$ 50,000.00 \text {. }\end{array}$ & Fair \\
\hline Hacialioglu et al. 2010 & Turkey & $\begin{array}{l}\text { Population: } 206 \text { caregivers of cancer patients } \\
\text { visiting a cancer center associated with a University } \\
\text { Hospital. Subjects were tiered in low, moderate, and } \\
\text { high income categories. } \\
\text { Assessment: WHOQOL - BREF TR }\end{array}$ & $\begin{array}{l}\text { Caregivers of lower-income status were observed } \\
\text { to share worse mean physical domain }(p=0.001) \text {, } \\
\text { social domain }(p=0.002) \text {, and national-environ- } \\
\text { mental }(p=0.043) \text { scores. }\end{array}$ & Fair \\
\hline Lukhmana et al. 2015 & India & $\begin{array}{l}\text { Population: } 200 \text { caregivers of cancer patients visiting } \\
\text { a University Hospital. Subjects were stratified by the } \\
\text { employment status of patients. } 103(51.5 \%) \text { patients } \\
\text { were unemployed and } 26(13 \%) \text { were laborers. } \\
\text { Assessment: ZBI }\end{array}$ & $\begin{array}{l}\text { Caregivers of unemployed patients }(p=0.007) \text {, } \\
\text { laborers }(p=0.043) \text {, and merchants }(p=0.046) \\
\text { shared greater burden than those caring for } \\
\text { semi-professional or professional patients. }\end{array}$ & Good \\
\hline
\end{tabular}

Nijboer et al. $2000 \quad$ Netherlands Population: 148 caregivers of cancer patients visiting 1 of 10 hospitals. Subjects were tiered in low, middle, and high socioeconomic categories. Subjects were evaluated at baseline, at 3 months, and at 6 months.

Assessment: CRA.

Oberst et al. $1989 \quad$ U.S.A. Population: 47 caregivers of cancer patients receiving radiotherapy through a cancer center in the Midwest region of the U.S.A. Subjects were tiered in lower, middle, and higher income categories. Assessment: ACS.

Caregivers of higher socioeconomic status were observed to share greater variability in burden during the course of providing aid compared to caregivers of lower socioeconomic status. These differences in variability were observed for the disrupted schedule $(\mathrm{p}<0.05)$, financial problem $(\mathrm{p}<0.05)$, and self-esteem $(\mathrm{p}<0.05)$ subscores.

Population: 330 caregivers of cancer patients visiting an urban regional cancer center. Subjects were tiered in higher and lower income categories.

Assessments: QOL (Family Version). Death anxiety was also assessed using the Temper Scale.

Lower-income caregivers shared worse threat Fair

ACS subscores compared to caregivers of higher social status. No differences were reported among the other subdivisions of the ACS based on socioeconomic status.

No difference in QOL was observed based on Fair socioeconomic status $(p=0.76)$. A trend towards worse QOL was observed among patients who required financial support compared to those who were financially-independent $(\mathrm{p}=0.06)$.

Vahidi et al. $2016 \quad$ Iran

Population: 150 male caregivers of breast cancer patients visiting a University Hospital. Subjects were tiered based on personal view of financial stability. Assessment: ZBI.

Caregivers who shared concerns regarding their living expenses reported greater caregiver burden $(p=0.019)$ than those who did not share financial stress.

Yang et al. $2012 \quad$ China

Population: 312 caregivers of cancer patients visiting a cancer center associated with a University Hospital. Subjects were stratified by monthly household income.

Assessment: CES-D

Depressive scores were higher among caregivers from homes earning less than 800 yuan $(\mathrm{p}<0.05)$, between 800 and 1499 yuan $(\mathrm{p}<0.05)$, and between 1500 and 1999 yuan $(\mathrm{p}<0.05)$ monthly compared to caregivers from homes earning more than 2000 yuan monthly.

Yoon et al. $2014 \quad$ South Population: 64 caregivers of terminally-ill cancer Korea patients admitted to a hospice-palliative care unit Subjects were stratified by monthly household income.

Assessment: CRA

Caregivers from homes earning less than KRW $2,000,000.00$ monthly were found to share worse disrupted schedule $(\mathrm{p}<0.05)$, family support $(\mathrm{p}<0.05)$, health problem $(\mathrm{p}<0.05)$, and financial problem $(p<0.05)$ subscores than those from homes earning more than KRW 2,000,00.00 monthly.

Yusuf et al. $2011 \quad$ Nigeria $\quad$ Population: 103 caregivers of cancer patients visiting a cancer center associated with a University Hospital. The mean annual income was equivalent to US $\$ 2616.00$.

Assessments: GHQ-30 and ZBI.

Caregivers who received financial support from relatives were observed to have better QOL per both assessments (GHQ-30, p $=0.001$; ZBI, $\mathrm{p}=0.001)$ compared to those who did not receive support. No differences were observed between employed and unemployed caregivers.

ACS, Appraisal of Caregiving Scale; CES-D, Center for Epidemiologic Studies Depression Scale; CRA, Caregiver Reaction Assessment Scale; GHQ-30, General Health Questionnaire; GSES, General Self-Efficacy Scale; QOL, Quality of Life; SF-12v2, Short Form Health Survey Version 2; WHOQOL - BREF TR, World Health Organization Quality of Life-Short Form, Turkish Version; ZBI, Zarith Burden Interview.

radiotherapy in the Midwest region of the U.S.A. using the Appraisal of Caregiving Scale (ACS) [19]. Worse scores were observed within the threat subdomain of the ACS for caregivers of lower socioeconomic status. Otherwise, the authors identified no differences in the harm/loss, challenge, and benign subdomains of the ACS based on socioeconomic status.

In summation, the available evidence suggests that lower-income caregivers of cancer patients in North America and Europe do not clearly share 
substantially worse QOL compared to caregivers of higher socioeconomic status. We believe lower-income caregivers within these countries may not experience worse QOL for two reasons. First, access to cancer care is more widespread for patients in the developed world. Consequently, quality of care may be reasonably equitable among rich and poor, thereby mitigating feelings of frustration among lower-income caregivers. Second, palliative care services are more available to lower-income patients in North America and Europe [24-25]. The burden of responding to emergent symptoms is often reduced with the aid of medical management directed towards improving QOL.

Lower-Income Cancer Caregivers in Africa, Asia, and the Middle-East

Three studies completed in the Middle East, 3 studies completed in South or East Asia, and 1 study completed in Nigeria were identified by systematic review [14, 15, 17, 20-23]. Hacialioglu et al. used the World Health Organization Quality of Life-Short Form, Turkish Version (WHOQOL - BREF TF) to evaluate QOL among family caregivers of Turkish patients actively undergoing chemotherapy [17]. Over half (57.5\%) of caregivers were described as lower-income though the authors did not report their actual earnings. The authors observed worse psychological, environment, and national subscores among lower-income caregivers. No differences were observed among varying levels of education. In Iran, Soleimani et al. used the Quality of Life (Family Version) scale to evaluate outcomes among caregivers for cancer patients undergoing treatment planning [20]. A significant population (30.9\%) of caregivers earned income within the poverty range for Iran. Though no differences in QOL were observed between levels of income, caregivers who relied on financial support from other family relatives reported worse QOL compared to those who were financially-independent. Similarly, Vahidi et al. reported that caregivers in Iran who have financial stress share worse ZBI scores than those who have more financial flexibility [21].

Both selected studies that were conducted in East Asia reported worse QOL among lower-income caregivers [15-22]. Yang et al. assessed depressive symptoms by administering the Center for Epidemiologic Studies Depressive Scale (CES-D) to caregivers for patients actively receiving chemotherapy for breast cancer, lung cancer, gynecologic cancer, or leukemia [22]. Caregivers who earned less than 800 Yuan (\$116.46 US) monthly shared the highest depressive scores. Similarly, Yoon et al. found greater burden among lower-income South Korean caregivers for terminally-ill cancer patients by administering the CRA [15]. Caregivers who earned less than 2,000,000 KRW (\$1790.75 US) monthly shared worse scores for all 4 subdomains of the CRA compared to those who earned more than 2,000,000 KRW monthly. In India, Lukhmana et al. compared ZBI scores among cancer caregivers of varying employment status [14]. Worse ZBI scores were observed for unemployed caregivers and caregivers with professions that required less education. The authors did not compare ZBI scores based on household income. Lastly, Yusuf et al. assessed burden among caregivers of Nigerian patients using the General Self-Efficacy Scale (GSES) and ZBI [23]. The mean annual income of the included subjects was equivalent to $\$ 2616.00$ US. The authors observed no differences in assessment scores between employed and unemployed caregivers; however, caregivers who received financial support from family relatives were found to have better QOL with both assessments compared to those who received no support.

Overall, the above studies reflect that lower-income caregivers in Asia and the Middle-East face a greater decline in QOL compared to those with more financial flexibility. The specific components of QOL that suffered most were difficult to identify because of a wide array of standardized assessments used by the selected studies. Nonetheless, 4 studies indicated that lower-income caregivers residing in Asia or the Middle-East commonly struggle with increasing burden [14-15-21, 23]. These findings are in accord with the results of our single-institutional study. Among African caregivers, limited data is currently available to determine the association between QOL and socioeconomic status.

\section{Implications for Practice and Future Studies}

The most salient finding from our cross-sectional study and systematic review is that caregivers of lower socioeconomic status in the developing world are at high-risk for a reduction in QOL. The burden associated with cancer caregiving is predominantly overwhelming. Clinicians who care for lower-income cancer patients, particularly in the Middle East and Asia, should be wary of the toll absorbed by the patients' caregivers. QOL among lower-income caregivers is more likely to deteriorate as the duration of providing aid to cancer patients extends.

Moderate success has been observed for interventions designed to improve caregiver QOL regardless of socioeconomic status [26]. Implementing these interventions, which often include counseling, exercise sessions, and/or interactive discussions, may be difficult in lower-income regions because of limited available resources. Consequently, we recommend two approaches to alleviate caregiver burden. First, educating and empowering relatives at the time of diagnosis to proportionally share the responsibility of caring for a lower-income cancer patient may be a resourceful method to maintain a balanced QOL for all involved. Second, since palliative care specialists are often not available, implementing departmental prescription protocols could help manage the cumbersome symptoms of advanced cancer that unfortunately arise and burden both patients and caregivers [27]. Such approaches may not carry significant financial costs but could prove significantly beneficial to lower-income cancer caregivers. 


\section{References}

1. U. Stenberg, C. M. Ruland, and C. Miaskowski, "Review of the literature on the effects of caring for a patient with cancer," (in eng), Psychooncology, vol. 19, no. 10, pp. 1013-25, Oct 2010.

2. M. I. Lapid et al., "Cancer caregiver quality of life: need for targeted intervention," (in eng), Psychooncology, vol. 25, no. 12, pp. 1400-1407, Dec 2016.

3. A. Girgis et al., "Some things change, some things stay the same: a longitudinal analysis of cancer caregivers' unmet supportive care needs," (in eng), Psychooncology, vol. 22, no. 7, pp. 1557-64, Jul 2013.

4. S. D. Lambert et al., "The unmet needs of partners and caregivers of adults diagnosed with cancer: a systematic review," (in eng), BMJ Support Palliat Care, vol. 2, no. 3, pp. 224-30, Sep 2012.

5. Y. H. Yun et al., "Economic burdens and quality of life of family caregivers of cancer patients," (in eng), Oncology, vol. 68, no. 2-3, pp. 107-14, 2005.

6. E. Blackmon, J. Bell, R. L. Whitney, S. C. Reed, K. Kim, and J. Joseph, "Caregiver work modifications: A hidden cost of cancer care," vol. 34, no. 3 suppl, pp. 190-190, 2016.

7. V. Noronha, R. Pinninti, V. M. Patil, A. Joshi, and K. Prabhash, "Lung cancer in the Indian subcontinent," (in eng), South Asian J Cancer, vol. 5, no. 3, pp. 95-103, Jul-Sep 2016.

8. S. Mishra et al., "Trends in bidi and cigarette smoking in India from 1998 to 2015, by age, gender and education," (in eng), BMJ Glob Health, vol. 1, no. 1, p. e000005, 2016.

9. M. A. Weitzner, P. B. Jacobsen, H. Wagner, Jr., J. Friedland, and C. Cox, "The Caregiver Quality of Life Index-Cancer (CQOLC) scale: development and validation of an instrument to measure quality of life of the family caregiver of patients with cancer," (in eng), Qual Life Res, vol. 8, no. 1-2, pp. 55-63, 1999.

10. A. Lafaye, S. De Chalvron, N. Houede, H. Eghbali, and F. Cousson-Gelie, "The Caregivers Quality of Life Cancer index scale (CQoLC): an exploratory factor analysis for validation in French cancer patients' spouses," (in eng), Qual Life Res, vol. 22, no. 1, pp. 119-22, Feb 2013.

11. D. Moher, A. Liberati, J. Tetzlaff, and D. G. Altman, "Preferred reporting items for systematic reviews and metaanalyses: the PRISMA statement," (in eng), Int J Surg, vol. 8, no. 5, pp. 336-41, 2010.

12. National Heart, Lung, and Blood Institute. Development and use of quality assessment tools. Available: www.nhlbi. nih.gov/health-pro/guidelines/in-develop/cardiovascularrisk-reduction/tools

13. W. D. Duggleby et al., "Hope of rural women caregivers of persons with advanced cancer: guilt, self-efficacy and mental health," (in eng), Rural Remote Health, vol. 14, p. 2561, 2014.

14. S. Lukhmana, S. K. Bhasin, P. Chhabra, and M. S. Bhatia, "Family caregivers' burden: A hospital based study in 2010 among cancer patients from Delhi," (in eng), Indian J Cancer, vol. 52, no. 1, pp. 146-51, Jan-Mar 2015.

15. S. J. Yoon, J. S. Kim, J. G. Jung, S. S. Kim, and S. Kim, "Modifiable factors associated with caregiver burden among family caregivers of terminally ill Korean cancer patients," (in eng), Support Care Cancer, vol. 22, no. 5, pp. 1243-50, May 2014.

16. N. E. Goldstein, J. Concato, T. R. Fried, S. V. Kasl, R. Johnson-Hurzeler, and E. H. Bradley, "Factors associated with caregiver burden among caregivers of terminally ill patients with cancer," (in eng), J Palliat Care, vol. 20, no. 1, pp. 38-43, Spring 2004.

17. N. Hacialioglu, N. Ozer, E. Yilmaz Karabulutlu, N. Erdem, and B. Erci, "The quality of life of family caregivers of cancer patients in the east of Turkey," (in eng), Eur J Oncol Nurs, vol. 14, no. 3, pp. 211-7, Jul 2010.

18. C. Nijboer, M. Triemstra, R. Tempelaar, M. Mulder, R. Sanderman, and G. A. van den Bos, "Patterns of caregiver experiences among partners of cancer patients," (in eng), Gerontologist, vol. 40, no. 6, pp. 738-46, Dec 2000.

19. M. T. Oberst, S. E. Thomas, K. A. Gass, and S. E. Ward, "Caregiving demands and appraisal of stress among family caregivers," (in eng), Cancer Nurs, vol. 12, no. 4, pp. 20915, Aug 1989.

20. M. A. Soleimani, R. H. Lehto, R. Negarandeh, N. Bahrami, and Y. H. Chan, "Death Anxiety and Quality of Life in Iranian Caregivers of Patients With Cancer," (in eng), Cancer Nurs, vol. 40, no. 1, pp. E1-e10, Jan/Feb 2017.

21. M. Vahidi et al., "Other Side of Breast Cancer: Factors Associated with Caregiver Burden," (in eng), Asian Nurs Res (Korean Soc Nurs Sci), vol. 10, no. 3, pp. 201-206, Sep 2016.

22. X. Yang et al., "Factors related to depressive symptoms among Chinese caregivers of cancer patients," (in eng), Psychooncology, vol. 21, no. 10, pp. 1063-70, Oct 2012.

23. A. J. Yusuf, A. Adamu, and F. T. Nuhu, "Caregiver burden among poor caregivers of patients with cancer in an urban African setting," (in eng), Psychooncology, vol. 20, no. 8, pp. 902-5, Aug 2011.

24. R. S. Morrison, "A National Palliative Care Strategy for Canada," (in eng), J Palliat Med, vol. 21, no. S1, pp. S63-s75, Jan 2018

25. C. Centeno et al., "Facts and indicators on palliative care development in 52 countries of the WHO European region: results of an EAPC Task Force," (in eng), Palliat Med, vol. 21, no. 6, pp. 463-71, Sep 2007

26. L. L. Northouse, M. C. Katapodi, L. Song, L. Zhang, and D. W. Mood, "Interventions with family caregivers of cancer patients: meta-analysis of randomized trials," (in eng), CA Cancer J Clin, vol. 60, no. 5, pp. 317-39, Sep-Oct 2010.

27. D. Lamas and L. Rosenbaum, "Painful inequities--palliative care in developing countries," (in eng), N Engl J Med, vol. 366, no. 3, pp. 199-201, Jan 192012.

\section{(i) (8)}

This work is licensed under a Creative Commons AttributionNon Commercial 4.0 International License. 